\title{
Avaliação eletromiográfica e ultrassonográfica do músculo masseter em indivíduos com paralisia facial periférica unilateral
}

\section{Electromyographic and ultrasonographic evaluation of the masseter muscle individuals with unilateral peripheral facial paralysis}

\author{
Fernanda Chiarion Sassi', Laura Davison Mangilli ${ }^{2}$, Danilo Pacheco de Queiroz ${ }^{3}$, Raquel Salomone ${ }^{4}$, \\ Claudia Regina Furquim de Andrade 5 .
}

1) Doutora em Ciências pela Faculdade de Medicina da Universidade de São Paulo. Fonoaudióloga do Departamento de Fisioterapia, Fonoaudiologia e Terapia Ocupacional da Faculdade de Medicina da Universidade de São Paulo.

2) Doutoranda do Programa de Ciências da Reabilitação do Departamento de Fisioterapia, Fonoaudiologia e Terapia Ocupacional da FMUSP. Fonoaudióloga do Instituto Central do Hospital das Clínicas da Faculdade de Medicina da Universidade de São Paulo.

3) Mestrando do Programa de Ciências da Reabilitação do Departamento de Fisioterapia, Fonoaudiologia e Terapia Ocupacional da Faculdade de Medicina da Universidade de São Paulo.

4) Doutoranda em Otorrinolaringologia pelo Hospital das Clínicas da Faculdade de Medicina da Universidade de São Paulo. Colaboradora do Ambulatório de Paralisia Facial Periférica do Hospital das Clínicas da Faculdade de Medicina da Universidade de São Paulo.

5) Professora Titular do Departamento de Fisioterapia, Fonoaudiologia e Terapia Ocupacional da Faculdade de Medicina da Universidade de São Paulo.

Instituição: Departamento de Fisioterapia, Fonoaudiologia e Terapia Ocupacional da Faculdade de Medicina da Universidade de São Paulo. São Paulo/SP - Brasil.

Endereço para correspondência: Claudia Regina Furquim de Andrade - Rua Cipotânea, 51- Cidade Universitária - São Paulo / SP - Brasil - CEP: 05360-160 - Telefone: (+55 11) 3091-8406 - Fax: (+55 11) 3091-7714 - E-mail: clauan@usp.br

Artigo recebido em $1^{\circ}$ de Julho de 2011. Artigo aprovado em 31 de Julho de 2011.

\section{RESUMO}

Introdução: Indivíduos com paralisia facial (PF) periférica têm condições que induzem à mastigação unilateral, realizada pelo lado não afetado, principalmente pela dificuldade de ação do músculo bucinador.

Objetivo: Caracterizar o controle motor e a morfologia do músculo masseter em indivíduos com PF periférica unilateral, através da avaliação eletromiográfica e ultrassonográfica.

Método: 16 participantes, de ambos os gêneros, com idade superior a 18 anos. O grupo pesquisa (GP) foi constituído de oito indivíduos com $\mathrm{PF}$ periférica unilateral idiopática a mais de seis meses; e o grupo controle (GC) por oito indivíduos normais. Todos os sujeitos foram submetidos à avaliação do músculo masseter através da Eletromiografia de Superfície (EMGs) e da Ultrassonografia (USG), nas seguintes tarefas: repouso, apertamento dentário com rolete de algodão entre os dentes (AL) e apertamento dentário com máxima intercuspidação dentária (MIC).

Resultados: Não houve diferença estatisticamente significante nas comparações intra e inter grupos quanto à assimetria entre as hemifaces, tanto para a EMGs quanto para a USG. Também não foram encontradas diferenças significantes entre a ativação dos músculos mastigatórios (masseter e temporal) na EMGs. Conclusão: $O$ controle motor e a morfologia dos músculos masseteres em indivíduos com PF periférica unilateral apresentam-se de forma semelhante aos indivíduos normais. Apesar da literatura sugerir que a demanda das adaptações funcionais realizadas por indivíduos com PF poderiam exceder a tolerância estrutural e funcional das articulações temporomandibulares, os resultados encontrados indicam que o tempo da PF dos pacientes estudados não foi suficiente para gerar diferenças anatômicas e fisiológicas nos músculos mastigatórios.

Palavras-chave: paralisia facial, eletromiografia, ultrassonografia, músculo masséter, avaliação.

\section{SUMMARY}

Introduction: Individuals with peripheral Facial Paralysis (FP) show conditions that lead to unilateral mastication, performed by the non-affected side, mainly due to the difficulty of action of the buccinator muscle.

Objectives: characterize the motor control and morphology of the masseter muscle in individuals with unilateral peripheral FP through electromyographic and ultrasonographic evaluation.

Method: 16 participants, of both sexes, with ages superior to 18 years old. The study group (SG) consisted of 8 individuals who'd had idiopathic unilateral peripheral FP for more than 6 months; the control group (CG) consisted of 8 normal individuals. All the subjects were submitted to the masseter muscle evaluation through surface electromyography (sEMG) and ultrasonography (USG) during the following tasks: rest, clenching with cotton roller between the teeth (CT) and clenching with maximum intercuspation (MIC).

Results: There was no statistically significant difference in comparisons within and between the groups concerning the hemifacial asymmetry, both for the sEMG and for the USG. Also there were no significant differences in the activation of the masticatory muscles (masseter and temporal) in the sEMG. Conclusions: Both the motor control and the morphology of the masseter muscles in individuals with unilateral peripheral FP were similar to those of normal individuals. Although literature suggests that the demand of functional adaptations made by FP individuals could exceed the structural and functional tolerance of the temporomandibular joints, the results indicate that the length of analyzed patient's FP was not enough to generate anatomical and physiological differences in the masticatory muscles.

Keywords: facial paralysis, electromyography, ultrasonography, masseter muscle, evaluation. 


\section{INTRODUÇÃO}

A paralisia facial é referida como a interrupção da informação motora para a musculatura facial. A Paralisia de Bell caracteriza-se como uma paralisia facial periférica do tipo idiopática, geralmente unilateral e de início súbito, através de um acometimento no nervo facial (VII par craniano) de forma aguda (1).

A paralisia dos músculos da mímica facial acarreta distúrbios funcionais e estéticos, além de gerar dificuldades sociais, psicológicas e/ou profissionais (2,3). A falta de movimentos e de expressão de um dos lados da face, assim como as alterações no modo de falar e se alimentar, constituem uma das alterações mais evidentes (4).

Indivíduos com paralisia facial periférica potencialmente têm condições que induzem à mastigação unilateral, realizada pelo lado não afetado, principalmente pela dificuldade de ação do músculo bucinador, uma vez que com a pouca participação deste músculo, há acúmulo de resíduos na região de vestíbulo oral do lado afetado (3).

Na mastigação unilateral, a musculatura mastigatória, especialmente dos músculos masseter, temporal e bucinador, apresenta maior funcionalidade do lado onde à mastigação está ocorrendo. A musculatura do lado contralateral encontra-se, via de regra, mais alongada e com tônus funcional diminuído, podendo até haver uma assimetria muscular visualmente perceptível $(3,5)$.

Em estudo anterior (6) verificaram diferença estatisticamente significante entre a amplitude da abertura da boca, lateralidade esquerda e protrusiva entre pacientes com Paralisia de Bell e indivíduos normais. Com base nesses resultados, sugerem que a demanda das adaptações funcionais nos pacientes com Paralisia Facial de Bell possa estar excedendo a tolerância estrutural e funcional das Articulações Temporomandibulares (ATMs), favorecendo o aparecimento de sinais compatíveis a quadro de disfunção temporomandibular.

A Eletromiografia de Superfície (EMGs) tem sido empregada por pesquisas que se relacionam à avaliação da função mastigatória, sendo um método válido para a avaliação dos músculos mastigatórios, podendo ser correlacionada à eficiência mastigatória $(7,8)$.

A EMGs é um exame indolor e não invasivo, e permite o estudo da atividade muscular, possibilitando a captação dos potenciais de ação gerados durante a contração dos músculos, os quais podem ser analisados considerando-se os parâmetros duração e amplitude $(3,4,9,10)$.
A Ultrassonografia apresenta vantagens consideráveis sobre outras formas de avaliação por imagem, como os exames de tomografia computadorizada e ressonância magnética, o que a torna um método mais apropriado para estudos realizados em larga escala. Em comparação à tomografia computadorizada, a Ultrassonografia não apresenta efeitos biológicos cumulativos conhecidos. É um método simples e de baixo custo para medir a espessura de músculos, desde que o radiologista siga um protocolo específico $(11,12)$.

A Ultrassonografia utilizada com o objetivo de medir os cortes musculares pode se relacionar a algumas patologias e a força muscular, além de outros parâmetros relacionados à funcionalidade e a idade (13-16). Porém a significância da Ultrassonografia como técnica reprodutível na avaliação do músculo masseter ainda é incerta e os índices de confiabilidade encontrados na literatura são bastante variáveis (17)

Com o intuito de verificar implicações funcionais da paralisia facial na biomecânica das ATMs, o presente estudo teve como objetivo caracterizar o controle motor e a morfologia dos músculos masseteres em indivíduos com paralisia facial periférica unilateral, através da avaliação eletromiográfica de superfície e ultrassonográfica.

\section{MÉTODO}

Participaram da pesquisa 16 indivíduos, de ambos os gêneros, com idade superior a 18 anos. O grupo pesquisa (GP) foi constituído por 8 indivíduos com paralisia facial (PF) periférica unilateral, 4 do gênero masculino e 4 do gênero feminino (idade média 37.8 anos); e o grupo controle (GC) por 8 indivíduos saudáveis pareados por gênero e idade ao GP (idade média 35.9 anos).

Os procedimentos de seleção e avaliação dos participantes só foram iniciados após os processos éticos pertinentes: parecer da comissão de ética (CAPPesq HCFMUSP no 0686/09 - 24/02/2010) e assinatura do Termo de Consentimento Livre e Esclarecido.

\section{Critérios de inclusão de GP}

Foram incluídos no GP indivíduos com idade igual ou superior a dezoito anos, que apresentavam:

a. Diagnóstico registrado em prontuário médico de PF, de origem periférica, idiopática, sem intervenção cirúrgica de reconstrução ou reanimação do nervo facial.

b. Paralisia facial há mais de seis meses.

c. Ausência de histórico de traumas de face e/ou cirurgias em região facial ou cervical. 
d. Ausência do uso de próteses dentárias, parcial ou total.

e. Ausência de acompanhamento fonoaudiológico prévio.

f. Pontuação entre quatro e onze no Protocolo de Avaliação Clinica da Mímica Facial do Lado Paralisado (18).

\section{Critérios de inclusão de GC}

Foram incluídos no GC indivíduos com idade igual ou superior a dezoito anos, que não apresentavam:

a) Queixa ou diagnóstico médico de paralisia facial.

b) Alterações no Sistema Estomatognático e em região de cintura escapular.

c) Alteração na dentição permanente (podendo ser aceita a ausência/extração dos $3^{\circ}$ molares) e alteração no padrão facial.

d) Uso de aparatologia ortodôntica.

e) Tratamento fonoaudiológico prévio.

f) Histórico de traumas de face e/ou cirurgias em região facial ou cervical;

g) Uso de próteses dentárias, parcial ou total.

h) Pontuação de 19 ou 20 no Protocolo de Avaliação Clinica da Mímica Facial (18).

A metodologia e procedimentos adotados para a avaliação dos participantes deste estudo encontram-se descritos abaixo:

\section{Eletromiografia de superfície}

Para a avaliação eletromiográfica foi utilizado o equipamento Miotool 400 (Miotec Equipamentos Biomédicos) com 4 canais, calibrados em 500 microvolts $(1 / 4 \mathrm{~V})$ com filtro do tipo passa banda $(20-500 \mathrm{~Hz})$ e ganho de 100 vezes, com baixo nível de ruído $(<5 \mu \mathrm{V}$ RMS considerado pela Intercational Society of Electrophysiology and Kinesiology - ISEK (1999) (19) como o recomendável.

O software utilizado para a captação e processamento do exame de eletromiografia de superfície foi o aplicativo Miograph 2.0 do mesmo fabricante, que realiza a aquisição, armazenamento e processamento on-line de sinais executado sob o sistema operacional Windows XP.

Os sinais da atividade elétrica dos movimentos musculares foram captados por eletrodos bipolares de superfície de Ag/AgCl, descartáveis, modelo SDS500, fixados com fita transpore (3M).

O posicionamento dos eletrodos obedeceu à técnica de colocação do ponto médio do ventre muscular na direção longitudinal do feixe muscular na posição mesodistal do músculo sugerido por estudo anterior (20) onde se observa maior amplitude de sinal para este tipo de eletrodo. Para garantir o correto posicionamento dos eletrodos inicialmente foi realizado a identificação dos músculos por meio da palpação durante o repouso e a contração máxima - foi solicitada a máxima intercuspidação dentária. Após esta etapa a função muscular foi testada para a verificação de possíveis erros de posicionamento e realizada nova colocação do eletrodo, se necessário.

Foi avaliada a atividade elétrica dos músculos temporais e masseteres $(10,21)$ durante:

- Repouso.

- Apertamento dentário com rolete de algodão entre os dentes (AL).

- Apertamento dentário com máxima intercuspidação dentária (MIC).

Para a coleta os participantes estavam confortavelmente sentados em uma cadeira, com as costas apoiadas, pés apoiados no chão, mãos apoiadas nos membros inferiores, cabeça posicionada adequadamente (Plano de Frankfurt paralelo ao chão), olhos abertos e buscando um ponto fixo pré-determinado. Todos os indivíduos foram orientados quanto ao teste. A pele da face foi limpa utilizando gaze embebida em álcool 70\% para remover a oleosidade, favorecendo a impedância. Os sinais captados foram analisados em RMS e expresso em microvolts $(1 / 4 \mathrm{~V})$.

O cabo de referência (cabo terra) foi conectado a e eletrodo fixado sobre o pulso, por ser uma região distante e neutra em relação aos músculos que foram analisados.

Primeiramente foi realizada a coleta dos músculos em repouso, durante 30 segundos. Foram realizadas três coletas e obtida a média da atividade elétrica em repouso destes músculos.

Em seguida, foi solicitado que o participante permanecesse em repouso por 15 segundos, sem gravação. Após esse comando, foi colocado um rolete de algodão de 10 mm entre os primeiros e segundos molares bilateralmente, e solicitado que o participante mordesse com a máxima força possível o algodão por 5 segundos, três vezes seguidas, com intervalo de 5 segundos entre elas. Foram realizadas três coletas desta prova e obtida a média da atividade elétrica no apertamento dentário máximo com algodão (total de nove amostras de atividade elétrica de cada músculo).

O mesmo procedimento foi realizado para a obtenção do registro da atividade elétrica dos músculos masseteres em máxima intercuspidação dentária. 
Os sinais eletromiográficos foram registrados e analisados posteriormente. Os traçados eletromiográficos foram obtidos em sinal bruto (RAW) e analisados em sinal retificado (RMS).

\section{Análise dos resultados eletromiográficos}

Para a análise dos resultados da EMGs foi utilizado a análise do domínio temporal. Neste caso, a informação obtida descreve em que momento o evento ocorreu e qual a amplitude (indicador da magnitude da atividade muscular) de sua ocorrência. Na situação de repouso, os valores obtidos representaram a média (RMS) da atividade eletromiográfica observada em 30 segundos. A duração da atividade muscular durante as tarefas de apertamento dentário (Al e MIC) foi obtida pela seleção do trecho representativo da ativação muscular (situação on, picoe off). Esse trecho foi selecionado com o cursor do próprio programa de eletromiografia e convertido em $\mu \mathrm{V}$ (Figura 1).

\section{Confiabilidade}

Com base na literatura relacionada, que aponta subjetividade na leitura das medidas da EMGs, foi realizada análise de confiabilidade a fim de determinar o índice de concordância entre os examinadores e assim garantir maior fidedignidade das medidas. Para tanto, foram selecionadas randomicamente 14 amostras eletromiográficas de um total de 144 . Essas amostras foram analisadas, independentemente, por dois pesquisadores independentes com experiência na área. O coeficiente de correlação mostrou-se alto para todas as comparações (intervalo de confiança de $95 \%$ [IC] $=0.9245-0.9567$ ), indicando alta consistência entre os examinadores.

\section{Ultrassonografia}

A avaliação da espessura do músculo masseter foi realizada utilizando a metodologia proposta por estudo anterior (14). Todas as imagens foram realizadas pelo mesmo radiologista utilizando o equipamento Philips L125/MSK Gen.

Durante a obtenção das imagens, o transdutor foi posicionado perpendicularmente à superfície da pele, evitando-se pressionamento excessivo. As medidas foram obtidas na porção mais volumosa do masseter, próximo ao plano oclusal, aproximadamente no centro da região médiolateral da distância do ramo.

As imagens e medidas foram realizadas bilateralmente com os indivíduos em posição de supino em três

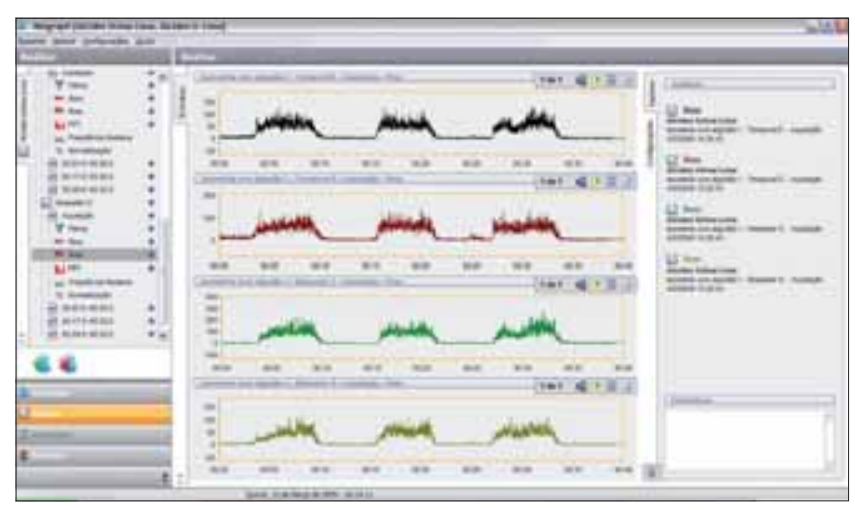

Figura 1. Atividade eletromiográfica em RMS dos músculos temporais e masseteres em máxima intercuspidação dentária com roletes de algodão.

condições diferentes: em repouso (posição habitual) (Figura 2); em máxima intercuspidação dentária com roletes de algodão de $10 \mathrm{~mm}$ entre as arcadas dentárias em região de primeiro e o segundo molares (Figura 3); e em máxima intercuspidação dentária sem os roletes de algodão (Figura 4).

As medidas foram realizadas no momento da coleta da imagem em tempo real. As imagens e medidas foram realizadas por três vezes, com um intervalo de cinco minutos entre cada medida.

\section{RESULTADOS}

Para as múltiplas comparações foi utilizado o Wilcoxon Matchedpairs test, com nível de significância de 5\%. Os resultados estão apresentados nas Tabelas 1, 2 e 3 .

Conforme observado nas Tabelas 1, 2 e 3, não houve diferença estatisticamente significante nas comparações intragrupos em todas as situações testadas, ou seja, ambos os grupos não apresentaram diferenças entre as hemifaces quanto à amplitude do sinal eletromiográfico e a espessura muscular em nenhuma das tarefas testadas. Além disso, não foi observada diferença estatística para a amplitude eletromiográfica entre os músculos mastigatórios (temporal x masseter) para ambos os grupos.

Para a comparação entre os grupos Na foi utilizado o teste de Mann-Whitney com nível de significância de 5\%. Nesta análise foram comparados os índices de assimetria facial de ambos os grupos (GP - Lado paralisado/Lado não paralisado; GC - Lado direito/lado esquerdo) para os resultados do músculo masseter e temporal na EMGs e do músculo masseter no USG (Tabelas 4 e 5). 
Conforme observado nas Tabelas 4 e 5 , não houve diferença estatisticamente significante nas comparações entre os grupos em todas as situações testadas, ou seja, os índices de assimetria entre as hemifaces foi semelhante para ambos os grupos.

\section{DISCUSSÃO}

Os resultados do estudo apontam que o controle motor e a morfologia dos músculos masseteres em indivíduos com paralisia facial periférica unilateral apresentamse de forma semelhante aos indivíduos normais.

A caracterização do controle motor dos músculos masseteres e temporais, avaliados através do Exame de EMGs, e da morfologia dos músculos masseteres, avaliada pelo Exame de USG, demonstrou não haver diferença estatisticamente significante entre os grupos, não havendo, portanto, uma diferenciação entre os mesmos.

Encontra-se na literatura que a EMGs pode ser influenciada pela espessura e camada de gordura na pele, posicionamento dos eletrodos e a motivação do paciente em realizar o mesmo (10). Estudos anteriores também discutem sobre a dificuldade em determinar, pelo exame de USG, se as mudanças na espessura muscular observadas são decorrentes da tarefa exigida ou de um erro de medição (8). Édefendido que o transdutor seja posicionado de forma padronizada e que o radiologista deva seguir um protocolo específico, como o realizado no presente estudo $(11,12)$.

Em estudo anterior (6) foi sugerido que a demanda das adaptações funcionais realizadas por indivíduos com Paralisia Facial de Bell, poderiam estar excedendo a tolerância estrutural e funcional das ATMs, favorecendo assim o aparecimento de sinais compatíveis a Quadro de Disfunção Temporomandibular. Outro estudo (22), onde foi realizada a avaliação do músculo masseter por meio da ressonância magnética, estabelecendo-se o índice da intensidade de

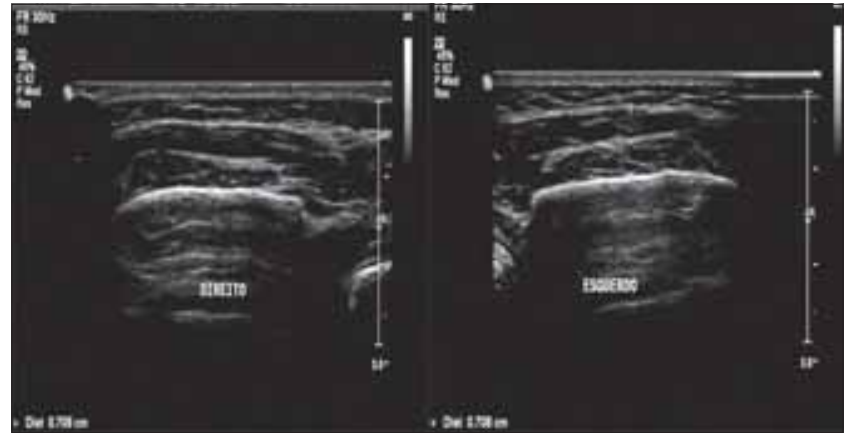

Figura 2. Imagem ultrassonográfica dos músculos masseteres direito e esquerdo no repouso.

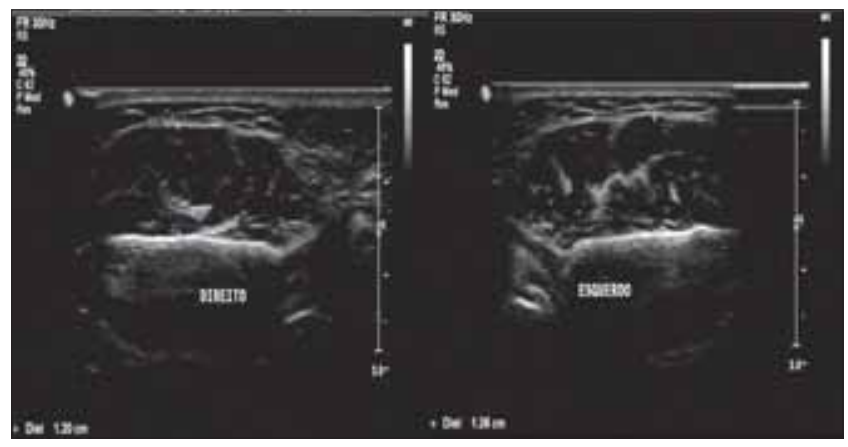

Figura 3. Imagem ultrassonográfica dos músculos masseteres direito e esquerdo em máxima intercuspidação dentária com roletes de algodão.

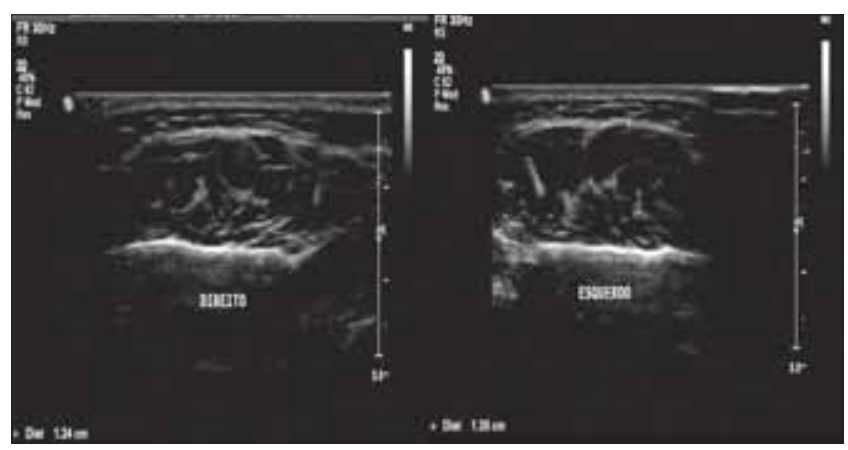

Figura 4. Imagem ultrassonográfica dos músculos masseteres direito e esquerdo em máxima intercuspidação dentária.

Tabela I . Assimetria entre as hemifaces - USG.

\begin{tabular}{lccccc}
\hline Grupo & Músculo & Condição & $T$ & $Z$ & p-level \\
\hline Controle & MExMD & Repouso & 15,500 & 0,350 & 0,726 \\
& & MIC & 17,000 & 0,140 & 0,889 \\
& & AL & 14,000 & 0,560 & 0,575 \\
Pesquisa & MPXMnP & Repouso & 12,000 & 0,840 & 0,401 \\
& & MIC & 17,000 & 0,140 & 0,889 \\
& & AL & 13,000 & 0,700 & 0,484 \\
\hline
\end{tabular}

Legenda: ME - Masseter esquerdo; MD - Masseter direito; MP - Masseter do lado paralisado; MnP - Masseter do lado não paralisado; MIC - Máxima intercuspidação dentária; AL - Máxima intercuspidação dentária com roletes de algodão. 
sinal do músculo masseter/substância branca, mostrou haver diferença estatisticamente significante entre indivíduos normais e indivíduos com Disfunção Temporomandibular.

O presente estudo não apresentou resultados que se assemelhassem aos estudos citados anteriormente. Acredita-se que o tempo de paralisia facial dos pacientes estudados não foi suficiente para gerar diferenças anatômicas e fisiológicas aos músculos mastigatórios avaliados em relação ao grupo de pacientes normais.

$\mathrm{Na}$ literatura direcionada ao estudo de alterações celulares que levariam à degeneração e atrofia de fibras musculares nos casos de paralisia facial, verifica-se que após aproximadamente vinte e quatro meses $(18,23,24)$ de alteração/lesão seriam verificados os primeiros sinais de atrofia e enrijecimento muscular.

Tabela 2. Assimetria entre as hemifaces - EMGs.

\begin{tabular}{|c|c|c|c|c|c|}
\hline Grupo & Músculo & Condição & $\mathrm{T}$ & Z & p-leve \\
\hline \multirow[t]{6}{*}{ Controle } & Temporal & Repouso & 16,000 & 0,280 & 0,779 \\
\hline & & MIC & 14,000 & 0,560 & 0,575 \\
\hline & & $\mathrm{AL}$ & | 4,000 & 0,560 & 0,575 \\
\hline & Masseter & Repouso & 15,000 & 0,420 & 0,674 \\
\hline & & MIC & | | ,000 & 0,980 & 0,327 \\
\hline & & $\mathrm{AL}$ & | I,000 & 0,980 & 0,327 \\
\hline \multirow[t]{6}{*}{ Pesquisa } & Temporal & Repouso & | 4,000 & 0,560 & 0,575 \\
\hline & & MIC & 10,000 & 1,120 & 0,263 \\
\hline & & $\mathrm{AL}$ & 17,000 & 0,140 & 0,889 \\
\hline & Masseter & Repouso & 16,000 & 0,280 & 0,779 \\
\hline & & MIC & 13,000 & 0,700 & 0,484 \\
\hline & & $\mathrm{AL}$ & | 4,000 & 0,560 & 0,575 \\
\hline
\end{tabular}

Legenda: MIC - Máxima intercuspidação dentária; AL - Máxima intercuspidação dentária com roletes de algodão.

Tabela 3. Comparação entre os músculos mastigatórios (masseter e temporal)-EMGs.

\begin{tabular}{lcccc}
\hline Grupo & Condição & $T$ & $Z$ & p-level \\
\hline Pesquisa & Repouso & 16,000 & 0,280 & 0,779 \\
& MIC & 8,000 & 1,400 & 0,161 \\
& AL & 17,000 & 0,140 & 0,889 \\
Controle & Repouso & 15,000 & 0,420 & 0,674 \\
& MIC & $\mid 4,000$ & 0,560 & 0,575 \\
& AL & 15,000 & 0,420 & 0,674 \\
\hline
\end{tabular}

Legenda: MIC - Máxima intercuspidação dentária; AL - Máxima intercuspidação dentária com roletes de algodão.

Tabela 4. Assimetria entre os grupos - USG.

\begin{tabular}{lcccccccccc}
\hline & $\begin{array}{c}\text { RankSum } \\
\text { PF }\end{array}$ & $\begin{array}{c}\text { RankSum } \\
\text { Controle }\end{array}$ & $U$ & $Z$ & p-level & Zadjusted & p-level & $\begin{array}{c}\text { Valid } \\
\text { NPF }\end{array}$ & $\begin{array}{c}\text { Valid } \\
\text { NControle }\end{array}$ & $\begin{array}{c}\text { 2*Isided } \\
\text { exactp }\end{array}$ \\
\hline REP & 76,000 & 60,000 & 24,000 & 0,840 & 0,401 & 0,840 & 0,401 & 8 & 8 & 0,442 \\
MIC & 69,000 & 67,000 & 31,000 & 0,105 & 0,916 & 0,105 & 0,916 & 8 & 8 & 0,959 \\
AL & 72,000 & 64,000 & 28,000 & 0,420 & 0,674 & 0,420 & 0,674 & 8 & 8 & 0,721 \\
\hline
\end{tabular}

Legenda: REP - Repouso; MIC - Máxima intercuspidação dentária; AL - Máxima intercuspidação dentária com roletes de algodão. 
Tabela 5. Assimetria entre os grupos - EMGs.

\begin{tabular}{cccccccccccc}
\hline & & $\begin{array}{c}\text { RankSum } \\
\text { Pesquisa }\end{array}$ & $\begin{array}{c}\text { RankSum } \\
\text { Controle }\end{array}$ & $U$ & Z & p-level & Zadjusted & $\begin{array}{c}\text { p-level } \\
\text { ValidN }\end{array}$ & $\begin{array}{c}\text { ValidN } \\
\text { Pesquisa } \\
\text { Controle }\end{array}$ & $\begin{array}{c}\text { 2*ided } \\
\text { exactp }\end{array}$ \\
\hline REP & Temp & 66,000 & 70,000 & 30,000 & $-0,210$ & 0,834 & $-0,210$ & 0,834 & 8 & 8 & 0,878 \\
& Mass & 69,000 & 67,000 & 31,000 & 0,105 & 0,916 & 0,105 & 0,916 & 8 & 8 & 0,959 \\
MIC & Temp & 58,000 & 78,000 & 22,000 & $-1,050$ & 0,294 & $-1,050$ & 0,294 & 8 & 8 & 0,328 \\
& Mass & 73,000 & 63,000 & 27,000 & 0,525 & 0,600 & 0,525 & 0,600 & 8 & 8 & 0,645 \\
AL & Temp & 63,000 & 73,000 & 27,000 & $-0,525$ & 0,600 & $-0,525$ & 0,600 & 8 & 8 & 0,645 \\
& Mass & 74,000 & 62,000 & 26,000 & 0,630 & 0,529 & 0,630 & 0,529 & 8 & 8 & 0,574 \\
\hline
\end{tabular}

Legenda: REP - Repouso; MIC - Máxima intercuspidação dentária; AL - Máxima intercuspidação dentária com roletes de algodão; Temp - Músculo temporal; Mass - Músculo masseter.

Acredita-se que os músculos mastigatórios, também sofreriam alterações, em longo prazo, devido às adaptações funcionais, uma vez que indivíduos com paralisia facial periférica potencialmente têm condições que induzem à mastigação unilateral, realizada pelo lado não afetado, principalmente pela dificuldade de ação do músculo bucinador $(3,5)$.

O período médio de paralisia dos pacientes estudados (6,5 meses) e de possíveis adaptações funcionais encontram-se abaixo do tempo apontado pelos estudos anteriores, o que confirma a hipótese levantada.

Quando o músculo não é utilizado ocorre degradação das proteínas contráteis ocorrendo: redução do número de miofibrilas e do calibre das fibras - o que chamamos de hipotrofia muscular (25) - e, em alguns casos, eventual substituição do músculo inteiro por tecido gorduroso e colágeno (18). Pode ocorrer em casos de imobilização, de algumas patologias neurológicas, de redução do suprimento sanguíneo por longos períodos, de magreza prolongada, de desnutrição e particularmente na denervação, levando até ao quadro de atrofia, que se caracteriza por uma hipotrofia acentuada (25).

Atrofia muscular pronunciada será evidente em todo indivíduo com acometimento dos neurônios inferiores ou dos nervos periféricos. Mesmo a atrofia muscular leve geralmente resulta em alguma perda de mobilidade ou de força dos músculos.

A atrofia muscular muitas vezes acaba predispondo ao desequilíbrio muscular, podendo acarretar algumas disfunções articulares. Articulações íntegras, ausência de dor e desconforto e uma alimentação adequada, são fatores indispensáveis e de extrema importância para a realização de um trabalho seguro na busca pelo reequilíbrio.

\section{CONCLUSÃO}

Tais resultados demonstram que ainda neste perío- do de PF não há um prejuízo na morfologia e no controle motor do músculo masseteres e temporais. Desta forma, acredita-se que deva ser realizada a orientação e direcionamento das Funções Estomatognáticas nestes indivíduos, a fim de prevenir possíveis adaptações funcionais que poderão gerar alterações no funcionamento das ATMs.

Sugere-se que mais estudos sejam realizados para que se possa definir o tempo de Paralisia Facial decorrido que gere o aparecimento de adaptações funcionais e modificações estruturais nos músculos mastigatórios e nos componentes intra articulares temporomandibulares.

\section{REFERÊNCIAS BIBLIOGRÁFICAS}

1. Goffi-Gomez MVS, Vasconcelos LGE, Bernardes DFF. Intervenção Fonoaudiológica na Paralisia Facial. In: Leslie Piccolotto Ferreira. (Org.). Tratado de Fonoaudiologia. 1 ed. São Paulo: Roca; 2004. p.512-526.

2. Diels HJ. Facial paralysis: is there a role for a therapist? Facial Plast Surg. 2000, 16(4):361-4.

3. Rahal A, Goffi-Gomez MVS. Avaliação eletromiográfica do músculo masseter em pessoas com paralisia facial periférica de longa duração. Rev. CEFAC. 2007, 9(2):207-12.

4. Bernardes DFF, Goffi-Gomez MVS, Bento RF. Eletromiografia de superfície em pacientes portadores de paralisia facial periférica. Rev. CEFAC. 2009, 12(1):91-96.

5. Douglas CR. Fisiologia do ato mastigatório. In: Patofisiologia oral: fisiologia normal e patológica aplicada e odontologia e fonoaudiologia. São Paulo: Pancast; 1998. p. $245-72$.

6. Sassi FC, Mangilli LD, Poluca MC, Bento RF, Andrade CRF. Amplitude Mandibular em Pacientes com Paralisia Facial Periférica Idiopática. Brazilian journal of otorhinolaryngology (no prelo). 
7. Felício CM, Couto GA, Ferreira CLP, et al. Reliability of masticatory efficiency with beads and correlation with the muscle activity. Pró-Fono Revista de Atualização Científica. 2008, 20(4):225-30.

8. Mangilli LD, Sassi FC, Sernik RA, Tanaka C, Andrade CRF de. Avaliação eletromiográfica eultrassonográfica do músculo masseter em indivíduos normais: estudo piloto. Pró-Fono Revista de Atualização Científica. 2009, 21(3):261-4.

9. Bianchini EMG. Mastigação e ATM: avaliação e terapia. In: Marchesan IQ. Fundamentos em fonoaudiologia: aspectos clínicos da motricidade oral. Rio de Janeiro: GuanabaraKoogan. 1998. p.37-49.

10. Nakata Y, Ueda HM, Kato $M$, et al. Change in stomatognathic function induced by orthognathic surgery in patients with mandibular prognathism. J Oral Maxillofac Surg. 2007, 65:444-451.

11. Esformes JI, Narici MV, Maganaris CN. Measurement of human muscle volume using ultrasonographic. European Journal of Applied Physiology. 2002, 87:90-92.

12. Emshoff R, Bertram S, Brandlmaier I, et al. Ultrassonographic assessment of local cross-sectional dimension of masseter muscle sites: a reproducible technique? Journal of Oral Rehabilitation. 2002, 29:10591062.

13. Benington PCM, GardenerJE, Hunt NP. Masseter muscle volume measured using ultrasonographic and its relationship with facial morphology. Eur J Ortho. 1999, 21:659-670.

14. Bertram S, Brandlmaier I, Rudisch A, et al. Cross-sectional characteristics of the masseter muscle: an ultrasonographic study. Int J Oral Maxillofac Surg. 2003, 32:64-68.

15. Emshoff R, Emshoff I, Rudisch A, et al. Reliability and temporal variation of masseter muscle thickness measurements utilizing ultrasonography. J Oral Rehabil. 2003, 30:1168-1172.
16. Satiroglu F, Arun T, Isik F. Comparative data on facial morphology and musle thickness using ultrasonography. Eur J Ortho. 2005, 27:562-567.

17. Emshoff R, Bertram S, Strobl H. Cross-sectional charactistics of muscle of the head and neck. Oral Surgery, Oral Medicine, Oral Pathology, Oral Radiology, and Endodontics. 1997, 87:93.

18. Toledo PN. Efeito da terapia miofuncional em pacientes com paralisia facial de longa duração associada à aplicação de toxina botulínica. 2007. Tese (Doutorado em PósGraduação em Cirurgia Plástica) - Faculdade de Medicina da Universidade de São Paulo.

19. Standards for Reporting EMG data. Journal of Electromyography and Kinesiology. Feb 1999, 9(1):III-IV.

20. Soderberg GL, Cook MT. Electromyography in biomechanics. Phys Ther. 1984, 64(12):1813-20.

21. Sforza C, Peretta R, Grandi G, et al. Soft tissue facial planes and masticatory muscle function in skeletal Class III patients before and after orthognatic surgery treatment. J Oral Maxillofac Surg. 2008, 66:691-698.

22. Nassri LFG, Abdala N, Szejnfeld J, Nassri MRG. Análise comparativa entre os a chados de ressonância magnética do músculo facial masseter em indivíduos com e sem disfunção temporomandibular: parte II. Rev Sul-Bras Odontol. 2009, 6(4):401-5.

23. Rubin LR. The anatomy of a smile: its importance in the treatment of facial paralysis. Plast Reconstr Surg. 1974, 53:384-7.

24. Baker DC. Facial paralysis. In: McCarthy JG, editor. Plast surgery. Philadehphia: W.B. Saunders Company; 1990, 3 : p.2237-319.

25. Guyton AC, Hall J E. Tratado de Fisiologia Médica. São Paulo: Manole. 2006. 